\title{
De la quantification comptable et financière dans le secteur public : promesses et usages de la gestion par les résultats
}

Quantifying public sector accounting and finance: myths and practices of public management by numbers

Yuri Biondi, Stéphanie Chatelain-Ponroy et Samuel Sponem

\section{OpenEdition \\ Journals}

Édition électronique

URL : http://journals.openedition.org/pmp/1532

DOI : $10.4000 /$ pmp. 1532

ISSN : 2119-4831

Éditeur

Institut de Management Public (IDPM)

Édition imprimée

Date de publication : 15 octobre 2008

Pagination : 113-125

ISSN : 0758-1726

Référence électronique

Yuri Biondi, Stéphanie Chatelain-Ponroy et Samuel Sponem, « De la quantification comptable et financière dans le secteur public : promesses et usages de la gestion par les résultats », Politiques et management public [En ligne], Vol. 26/3 | 2008, mis en ligne le 25 octobre 2011, consulté le 19 avril 2019. URL : http://journals.openedition.org/pmp/1532 ; DOI : 10.4000/pmp.1532 


\title{
DE LA QUANTIFICATION COMPTABLE ET FINANCIERE DANS LE SECTEUR PUBLIC : PROMESSES ET USAGES DE LA GESTION PAR LES RESULTATS
}

\author{
Yuri BIONDI ${ }^{1}$ \\ Stéphanie CHATELAIN-PONROY ${ }^{2}$ \\ Samuel SPONEM ${ }^{3}$
}

\begin{abstract}
Résumé Regroupées sous le label de "nouvelle gestion publique ", de nombreuses réformes des administrations publiques sont expérimentées depuis une trentaine d'années dans les pays de l'OCDE et ailleurs. Ces réformes se traduisent par la mise en œuvre d'instruments de gestion et sont porteuses de promesses de rationalisation, démocratisation et performance accrue.

Pourtant, comme le souligne l'OCDE dans une tentative de bilan partiel en 2005, "la réalité de la réforme n'a pas été à la hauteur du discours ". Les promesses non tenues suggèrent donc de questionner ces dispositifs afin d'en percevoir les rôles spécifiques dans les organisations et la société.

Cette compréhension avertie des dispositifs de gestion en situation éclaire leur impact sur les choix et les comportements et leur capacité à rendre possibles, voire nécessaires, des choix politiques qu'ils ne sauront remplacer.
\end{abstract}

\footnotetext{
${ }^{1}$ Cnrs (CRG - PREG, Ecole Polytechnique Paris) et Conservatoire National des Arts et Métiers

${ }^{2}$ Conservatoire National des Arts et Métiers, GREG (EA 2430) - Centre de recherche en comptabilité

${ }^{3}$ Conservatoire National des Arts et Métiers, GREG (EA 2430) - Centre de recherche en comptabilité Revue POLITIQUES ET MANAGEMENT PUBLIC, Volume 26, n³, 2008.

(C) Institut de Management Public - 2008.
} 
Introduction Les instruments de quantification comptable et financière sont au cœur des projets de réforme de la gestion publique qui touchent depuis de nombreuses années la plupart des pays de l'OCDE et notamment la France. Au-delà de leurs différences, liées aux contextes historique, culturel et géographique, ces dispositifs témoignent de la confiance qui est accordée aux chiffres depuis le début du XXème siècle (Porter, 1995 ) et sont porteurs de promesses communes relatives aux bienfaits de la gestion par les résultats. Ces promesses résistent-elles à l'épreuve des faits? Nous postulons que les déceptions apparues ici ou là sont en grand partie imputables à une vision réductrice des instruments de quantification, souvent présentés comme des moyens de rationalisation de la gestion publique et de démocratisation de l'action publique alors que leurs rôles sont plus variés et complexes qu'il n'y paraît de prime abord.

Nous présenterons, dans un premier temps, les promesses affichées par les discours porteurs de ces réformes, en nous attachant aux instruments comptables et financiers censés les mettre en œuvre. Nous nous efforcerons ensuite de situer ces dispositifs dans leur contexte organisationnel et sociétal, afin d'appréhender leurs rôles dans les organisations. Nous discuterons enfin du lien mécaniste, sous-jacent à la logique de contrôle à distance portée par ces dispositifs, entre financement et performance.

Les promesses de la « nouvelle gestion publique » : rationalisation de la gestion publique et démocratisation du débat public
Depuis une trentaine d'années, des réformes de la gestion publique sont mises en œuvre dans de nombreux pays de l'OCDE sous la pression de la crise économique, du poids de la dette ou de l'évolution des attentes des citoyens et des consommateurs. Ces réformes ont été rassemblées sous le vocable de "nouvelle gestion publique " dont on attend une amélioration de la performance des Etats et une rationalisation de leur gestion. Leur mise en œuvre passe souvent par le développement de nouveaux instruments comptables et financiers, facilitant une forme de gestion par les résultats, sans que cette évolution ne donne réellement lieu à débat.

En France, l'adoption de la Lolf a offert récemment un exemple de ce consensus puisque cette loi a fait l'objet d'un vote quasi-unanime du Parlement. Dans le domaine des instruments de quantification comptable et financière, la révision de la procédure de préparation du budget, la mise en place d'une comptabilité d'exercice à caractère patrimonial, les assouplissements en matière de gestion des crédits, la nouvelle architecture financière nous promettent une « révolution copernicienne " (Lambert, 2002) - dans la mesure où la gestion publique n'est plus centrée autour des moyens mais des résultats - et 
une gestion systématique de la performance, en d'autres termes : une gestion publique plus rationnelle.

De nombreux autres exemples de réformes comptables et financières pourraient être retenus. Parmi ceux-là, la rationalisation des choix budgétaires (RCB) des années 1970 qui avait pour objectif d'élaborer une procédure budgétaire permettant des décisions et des arbitrages rationnels et promettait une «rationalisation des choix budgétaires et un contrôle des résultats de l'action administrative » (décret du 25 mai 1970). A son tour, le "renouveau du service public ", apparu en 1989, promut les centres de responsabilité, les assouplissements des règles de gestion budgétaire et l'accroissement de l'autonomie administrative (circulaire du 23 février 1989) pour permettre une rationalisation de la gestion publique. Plus proche de nous, la révision générale des politiques publiques (RGPP), lancée le 10 juillet 2007, nous promet de "rendre l'administration plus efficace et d'améliorer les services rendus aux usagers " grâce à un « un Etat mieux géré, qui valorise le travail des fonctionnaires et qui utilise au mieux les ressources publiques " (rapport du ministre du budget, des comptes publics et de la fonction publique, décembre 2007).

Les instruments de quantification comptable et financière incarnent les nombreuses promesses de ces réformes relatives à l'amélioration de la performance publique par le progrès du service rendu au citoyen, la responsabilisation des ministres et des fonctionnaires et la comparaison avec le secteur privé (Trosa, 2000). Ces dispositifs sont ainsi mis au service d'une nouvelle forme de régulation de l'administration publique qui passe par la responsabilisation et le contrat managérial (Chaty, 1999). Ils devraient aussi faciliter l'évaluation et le contrôle de l'action publique, répondant ainsi à une exigence démocratique au travers de la clarification des débats politiques et sociaux (Club Cambon, 1989).

Les discours qui accompagnent leur mise en œuvre visent donc à convaincre de l'efficacité, de l'efficience et de la légitimité de ces processus de gestion par les résultats qui sont présentés comme des dispositifs incontournables de rationalisation et de démocratie, permettant tout à la fois d'établir des priorités, de rendre des comptes, de gérer à distance, de mesurer les réalisations, de justifier les choix et, finalement, d'améliorer les résultats de l'action publique. L'adoption de tels dispositifs est, en conséquence, présentée comme une évidente nécessité, une question technique dont il serait incongru de discuter le bien-fondé. 


\section{Une vision cybernétique des instruments de quantification comptable et financière}

L'étude des discours attachés aux différentes réformes montre que les instruments de quantification comptable et financière y sont toujours mobilisés dans une visée rationnelle, puisqu'ils doivent permettre un pilotage régulé et contrôlé des administrations liant mécaniquement priorités d'action, objectifs stratégiques, indicateurs de suivi, mesure des résultats et corrections éventuelles. L'adoption de ces dispositifs serait donc un gage d'amélioration de la rationalité, ce qui explique qu'ils se soient imposés comme des normes incontestables en matière de gestion publique : ils accompagnent alors une conception « instrumentale » de la gestion publique (Gibert, 2000) et sont réduits à leur dimension cybernétique permettant de gérer par les résultats.

Or, ces instruments, comme tous les dispositifs de gestion, sont composés "d'éléments matériels, humains et symboliques, en interaction systémique les uns avec les autres " (Boussard \& Maugeri, 2003). Les considérer comme de simples "machines à chiffrer », c'est en ignorer les rôles latents. En effet, selon Burchell et alii (1980), les représentations comptables et financières jouent dans la pratique des rôles divers en fonction des situations (voir tableau suivant).

Une vision cybernétique (case A) n'est envisageable que lorsque les objectifs politiques à atteindre et les activités pour les accomplir sont simples. Lorsque la mise en œuvre des politiques publiques est complexe et incertaine, les instruments comptables et financiers peuvent être utilisés pour apprendre, c'est-à-dire pour se poser des questions, mais ils ne peuvent être utilisés de manière mécanique pour atteindre les objectifs préfixés (case $B$ ). En outre, lorsque les objectifs mêmes de ces politiques sont complexes et incertains, les représentations comptables et financières peuvent être utilisées par chaque acteur pour favoriser ses intérêts (case $\mathrm{C}$ ), ou dans une optique de justification rhétorique (au nom de la rationalisation, ou de la démocratisation) des décisions prises (case D), ce qui peut masquer l'impact véritable des politiques adoptées.

Dimension politique : incertitude sur les objectifs

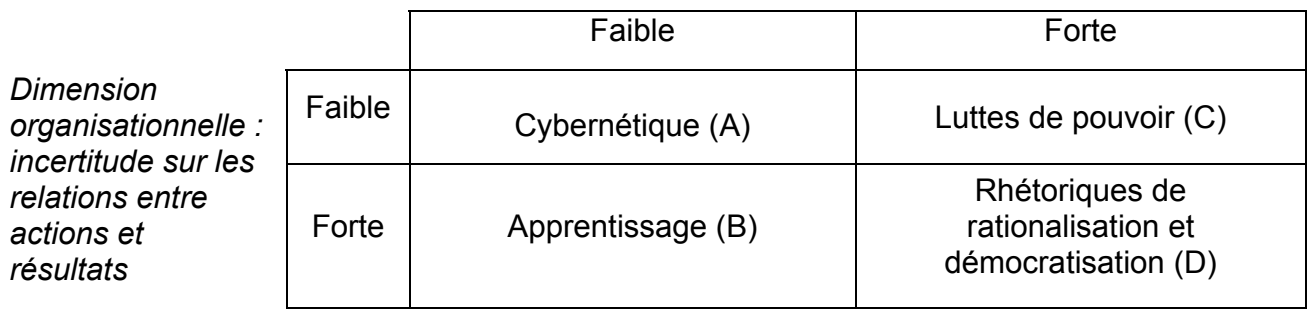

Tableau I - Les rôles de la quantification comptable et financière (adapté de Burchell et alii, 1980) 

promesses et usages de la gestion par les résultats

Cette prise en compte des rôles latents des instruments de quantification comptable et financière semble féconde pour mieux en comprendre les usages, aussi bien dans leur contexte organisationnel que dans leur dimension politique.

En ce qui concerne le contexte organisationnel (constitué des organisations qui doivent mettre en œuvre les politiques publiques), les modalités d'intervention sont toujours ambiguës, notamment du fait de la double "fonction de production » qui caractérise les biens publics (Gibert, 1986). La définition des modalités d'action est en effet particulièrement difficile, d'une part du fait de la complexité de ce que produit le secteur public (ce que l'on appelle les outputs, essentiellement constitués de services (Gadrey, 1991)) et, d'autre part, de l'impact de cette production sur la société (les outcomes) (Trosa, 2000). Dans ce cadre, la quantification peut aider le secteur public à appréhender l'impact de ses actions sur la société; elle joue alors un rôle d'apprentissage pour les organisations qui pourraient ainsi mieux comprendre et mettre en œuvre leurs missions (outcomes).

Par exemple, dans le cas d'un service public régalien tel que la justice, la quantification, en sa qualité de "machine à poser des questions" (Pallez, 2000), peut aider à réfléchir à l'allocation des ressources. En revanche, il serait illusoire d'essayer de l'utiliser pour mesurer la performance (outputs) de manière chiffrée, sous peine de forts effets pervers. Les mêmes difficultés se posent dans les secteurs de la police (Montjardet, 2006), la réinsertion des chômeurs (Knoepfel \& Varone, 1999), la santé, l'éducation ou encore la recherche (Weick 1976, Osterloh et alii, 2008). D'ailleurs, la simplification, par la quantification des outputs et outcomes de l'action de l'Etat peut aussi conduire à un appauvrissement de son action et à sa délégitimation (Knoepfel \& Varone, 1999).

Cette distinction résultats/externalités induit une séparation entre la dimension "productive » (organisationnelle) et la dimension " allocative » (sociale) qui ouvre à la dimension politique des instruments de quantification. En effet, l'usage de ces dispositifs conduit à définir, de facto, la performance ou, du moins, les axes sur lesquels celle-ci est mesurée. Or, cette définition, dans le secteur public plus qu'ailleurs, "fût-ce sous une forme édulcorée, est impossible à poser " (Gibert, 2000) car très largement conventionnelle (Gibert, 2002). Elle traduit, en effet, des choix politiques et permet de légitimer, en interne ou en externe, certaines pratiques (Burlaud \& Gibert, 1984).

La logique de gestion par les résultats peut donc conduire à la mise en œuvre de décisions politiques non explicitées: elle fait alors passer pour une mesure technique ce qui relève d'un choix politique. Par exemple, en matière de santé, "la méthode de calcul des coûts [...] définit ex-post un produit en lui adjoignant [...] un système de 'pseudo prix' ", ce qui peut conduire à " une spécialisation des activités [...], une sélection des malades, à des refus d'admission et à une diminution 
considérable de la durée moyenne de séjour » (Joncour, 1987). De surcroît, elle donne l'illusion de la bonne gestion pour tout ce qui est aisément quantifiable. Ainsi, un rapport parlementaire récent (Marini, 2007) constate que « les programmes les mieux pilotés [...] sont le plus souvent de nature financière, ce qui contribue à expliquer la qualité de leur gestion : ils sont plus faciles à gérer que, par exemple, des programmes portant sur la fourniture de services collectifs directement mis à la disposition du public, ou cherchant à agir sur la situation économique et sociale " ${ }^{4}$. La "bonne gestion » est ici évaluée par la capacité à quantifier les résultats de l'action publique. Au final, les méthodes de gestion favorisent certaines activités de l'Etat et définissent implicitement des orientations politiques à défaut de les énoncer clairement aux citoyens. Dans ce cas, peut-on réellement se prévaloir de l'apport de la quantification au débat démocratique?

Dans ce contexte, la quantification et, singulièrement, le recours à des dispositifs de gestion par les résultats, risque de renforcer les cercles vicieux bureaucratiques (Crozier, 1963) qu'elle était censée reformer. Certes, elle permet un apprentissage et un éclairage des objectifs visés mais elle risque, d'une part, d'imposer une logique cybernétique qui peut entamer le fonctionnement des organisations publiques et, d'autre part, de se substituer à la véritable fabrique démocratique des choix politiques.

Une vision sous-jacente de l'organisation
L'appréhension des usages et rôles latents des instruments comptables et financiers dans les organisations et la société met en exergue une perception "cybernétique " de ces dispositifs, dans une logique de " contrôle à distance » (Robson, 1992) qui suppose une séparation (bureaucratique) entre le choix politique et sa mise en œuvre (ChatelainPonroy, 2008).

En effet, l'empreinte "gestionnaire » des réformes en cours rend possible la délégation des services publics à des agences. L'introduction d'administrations publiques plus autonomes et décentralisées crée donc de véritables organisations qui réunissent choix politiques et organisationnels (gestionnaires) à un niveau plus opérationnel (Biondi, 2008). La performance de ces entités ne saurait résulter mécaniquement de mesures et responsabilités contrôlées à distance, mais passe par le fonctionnement même du système que constitue chaque entité.

\footnotetext{
${ }^{4}$ Tel est le cas des programmes 145 «Epargne », qui concerne les primes accordées par l'État dans le cadre des plans épargne logement (PEL), 119 "Concours financiers aux communes et groupements de communes ", qui concerne les dotations de l'État aux communes, 164 " Cour des comptes et autres juridictions financières », comprenant en particulier les crédits de la Cour des comptes.
} 
De la quantification comptable et financière dans le secteur public promesses et usages de la gestion par les résultats

C'est ainsi que la double question du contrôle, entre l'Etat et les agences d'une part et au sein même des agences d'autre part, se pose (Biondi, 2009). Le contrôle de cette organisation demande un « ordre » ordre de sens, d'action, de suivi - qui ne peut être celui d'une machine, mais celui de l'activité humaine et sociale représentée par chaque entité. Ce contrôle s'effectue et s'articule ainsi avec les modes de fonctionnement de l'organisation qui l'accueille et qui est en quête de légitimité, d'équité et d'efficacité. Les instruments comptables et financiers participent de cet ordre et touchent ses dimensions institutionnelle (gouvernance et régulation), organisationnelle (action et direction) et cognitive (connaissance et publication).

\begin{tabular}{|c|c|c|}
\hline Contrôle et « rendre compte » comme & Rôle de & Référence \\
\hline instrument cognitif & représentation ; suivi & le vrai \\
\hline instrument institutionnel & encadrement ; influence & le juste \\
\hline instrument organisationnel & direction ; incitation & la finalité \\
\hline
\end{tabular}

Tableau II - tiré de Biondi (2009)

En outre, cette vision cybernétique induit fréquemment la recherche d'un lien mécanique entre financement (de certaines politiques) et performance (de leur mise en œuvre), qui correspond à la vision mécaniste du contrôle de cette relation ${ }^{5}$. Or, malgré les attentes à ce sujet, l'introduction d'entités chargées d'accomplir des missions d'intérêt général n'assure pas, en elle-même, un niveau moins élevé des coûts. Au contraire, comme le montrent par exemple des études (OCDE) sur la dépense sanitaire (Montaguti \& Zanetti, 1993), les systèmes de gestion de la santé fondés sur des entités autonomes et quasi-autorégulées (en concurrence public-privé) ont certes la capacité d'améliorer la parité économique de chaque entité, mais engendrent, dans le même temps, un niveau de coût beaucoup plus élevé et une satisfaction des besoins moins étendue et équitable pour le système socio-économique dans son ensemble (un défaut éclatant de macro-régulation).

Dans ce contexte, quel rapport s'instaure entre financement et performance ? Rien de mécanique et de déterminé ne semble en effet conduire du financement des politiques publiques aux résultats obtenus par les organisations qui sont censées les accomplir. Notre analyse invite à distinguer les dimensions organisationnelle et sociopolitique des dispositifs de contrôle comptable et financier.

\footnotetext{
${ }^{5}$ La même vision mécaniste s'inscrit souvent dans la notion d'échange marchand contre un prix.
} 
Pour ce qui concerne la dimension organisationnelle, l'instrumentation de contrôle, si elle semble cohérente pour le calcul des coûts des activités, ne l'est pas pour l'accomplissement des finalités. Weick (1976) montre ainsi le découplage entre la mesure de la performance d'une organisation scolaire et le processus éducatif générateur de cette performance.

Ainsi, d'un point de vue financier, la solution simpliste d'une tarification de chaque service rendu semble engendrer des problèmes plutôt qu'offrir de véritables solutions. En effet, une fois que des contributions (impôts, transferts, participations aux frais) sont dépensées pour acquérir un matériel durable au sein de l'organisation, l'imputation de son coût aux usagers pourrait engendrer une réduction de son utilisation, alors même que la dépense est déjà faite, ce qui provoquerait un gaspillage. En outre, cette imputation pourrait ne pas être équitable, car le matériel ne doit pas forcément être payé par les usagers (ou les utilisations) dans une activité ou une organisation sans but lucratif. Enfin, elle pourrait compromettre l'accomplissement des finalités de l'organisation, car des activités importantes mais ne donnant pas lieu à des utilisations directes (ou massives) pourraient alors être négligées ou abandonnées. De plus, la mise en place de cette tarification pourrait déplacer l'attention organisationnelle (et l'emploi de ses ressources et de son personnel) vers ce processus d'encaissement et de contrôle plutôt que vers la qualité des services, et léser de ce fait la relation entre opérateurs et citoyens.

Pour ce qui concerne la dimension politique et sociétale, la mise en place d'un système cybernétique de gestion par les résultats semble entamer la marge d'autonomie et le principe de subsidiarité que les réformes affichent comme arguments de légitimation et d'efficacité. L'organisation des services publics concerne des aspects cruciaux de la vie démocratique d'une société, comme l'enseignement, la recherche, l'information. Dans ce contexte, la quantification, si elle n'améliore pas automatiquement la performance de ces organisations (au mieux, pourra-t-elle chiffrer les coûts), risque de les enfermer dans des luttes de pouvoir et des rhétoriques de justification dont les jeux organisationnels et institutionnels sont porteurs (cases $\mathrm{C}$ et $\mathrm{D}$ du tableau I).

Dans cette perspective, les réformes en cours gagneraient à délaisser les mesures de performance potentiellement contreproductives, pour appréhender différemment la légitimité, l'équité et l'efficacité organisationnelles qui peuvent ainsi être assurées (et contrôlées) par d'autres modalités, par exemple de style clanique, participatif ou politique (Ouchi, 1979; Hofstede, 1981). II ne s'agit donc plus de chercher à réduire en vain la complexité, ou d'arrêter la dynamique des activités organisationnelles par le biais de budgets et de mesures de performance financière, mais de laisser gérer ces organisations en situation, en faisant système entre choix politiques, modes de fonctionnement organisationnel, et modes de contrôle, s'éloignant ainsi 

questions.

Conclusion : le dépassement du mythe cybernétique
Comme toute évaluation, celle des résultats des réformes de la gestion publique est difficile (Trosa, 1992) et les tentatives d'évaluation sont, par conséquent, rares et ne permettent pas de dresser un bilan complet et définitif des actions de modernisation de la gestion publique. Cependant, la succession ininterrompue des réformes, leur abandon parfois (RCB...), leur similitude en dépit des contextes différents (historique, culturel, géographique...), la récurrence de certains thèmes, les tentatives de bilans partiels (Pollitt, 2002 ; Chemlay-Lafay \& Chol, 2006 ; OCDE, 2005) qui en ont été réalisées montrent que les objectifs ambitieux affichés et, en particulier, les promesses de rationalisation et démocratisation sont loin d'être tenus. Par exemple, Seddon (2008) souligne les gaspillages, augmentations de coûts, réductions de la qualité des services publics et destruction du moral et de la confiance du personnel et des citoyens usagers engendrés par les réformes anglaises.

Il existe ainsi des travaux de chercheurs qui s'efforcent de souligner les risques et les effets pervers de ces réformes et en montrent bien les limites. On sait, par exemple, que le recours à des instruments de mesure peut générer une masse d'information excessive ou inutile, qu'il peut inciter les acteurs à chercher à atteindre les objectifs qui leur sont fixés même au prix de conséquences néfastes sur leur activité, qu'il peut conduire à privilégier les améliorations visibles à court terme (ChatelainPonroy \& Sponem, 2008). On sait également que certaines dispositions des réformes inspirées par la nouvelle gestion publique peuvent mettre à mal les valeurs traditionnelles des administrations et ainsi conduire à de graves dysfonctionnements. On sait, enfin, que la succession de réformes comporte un risque important de démotivation et de manque d'adhésion de la part de ceux qui sont appelés à mettre en œuvre les réformes.

Finalement, ce que nous apprennent ces limites, c'est que le lien postulé par ces réformes entre le changement des instruments, celui des comportements et, au-delà, celui du mode de fonctionnement organisationnel, n'est ni automatique, ni univoque. Les travaux des chercheurs en comptabilité et contrôle de gestion ont montré depuis longtemps que les organisations ont une capacité à assimiler ou à détourner les instruments consacrés à leur maîtrise, sans modifier substantiellement leurs modes de fonctionnement (Bouquin, 2005) et que la promotion de mesures de résultat n'entraînait pas automatiquement la modification - pourtant recherchée - des 
comportements. En idéalisant les dispositifs au travers d'un mythe rationalisateur et en faisant porter leurs efforts sur eux seuls, les réformes mises en œuvre "n'ont pas abouti aux changements de comportement et de culture nécessaires à plus long terme " (OCDE, 2005). Autrement dit: pour qu'une réforme produise les effets qui en sont attendus, il faut passer d'un stade d'adoption des instruments celui où ces derniers sont réduits à leur dimension formelle, où la lettre de la réforme prime sur son esprit - à celui de leur appropriation (Chatelain-Ponroy, 2008).

On voit bien alors que le champ embrassé par les réformes de gestion publique devient autrement plus vaste et plus complexe que celui de leur simple adoption formelle de la part des administrations et des organisations publiques. C'est cette épreuve de la complexité qui explique, selon Matheson (2002), que si le lancement des réformes est politiquement rentable car synonyme de dynamisme et de modernité, leur mise en œuvre est, en revanche, très coûteuse et qu'elle échoue en général en raison des pierres d'achoppement que constituent les changements de comportements et de modes de fonctionnement.

L'ensemble de ces constats conduit à rechercher les moyens de " dépasser la simple mise en œuvre des dispositifs de gestion au nom de l'efficience et à envisager l'effet de ces instruments sur le dispositif gouvernemental considéré dans sa globalité afin de promouvoir la capacité du secteur public à s'adapter au changement tout en préservant ses valeurs essentielles » (OCDE, 2003).

L'appréhension de ces instruments dans leur contexte organisationnel montre qu'ils ne sont pas neutres, mais porteurs d'une vision. S'ils constituent le reflet d'un idéal de réalité, ils participent également à la transformation de cette réalité. C'est pourquoi il convient d'examiner attentivement leurs rôles dans les organisations qui mettent en œuvre les politiques publiques comme dans la société qui est censée en bénéficier. Ils peuvent y assumer un rôle d'apprentissage, puisqu'ils sont capables de représenter et alors d'éclairer un aspect ou une dimension spécifiques. Mais ils ne sauraient remplacer les questionnements des choix politiques qu'ils rendent ainsi possibles, voire nécessaires. 


\section{BIBLIOGRAPHIE}

BIONDI Y. (2009), « Du financement au contrôle de l'entité entreprise : une réflexion à partir de la tradition en comptabilité dynamique » en $\mathbf{L a}$ genèse de la décision. Chiffres publics, chiffres privés dans la France du XXe siècle, Philippe Verheyde, Béatrice Touchelay dir., Paris, éditions Biere.

BIONDI Y. (2008), « De Charybde de la comptabilité de caisse en Scylla de la comptabilité patrimoniale, ou du périlleux périple de la comptabilité de l'Etat en France (et ailleurs) ", Revue de la régulation, $n^{\circ} 3 / 4,2^{\text {ème }}$ semestre - Dossier « Normes et Institutions de la Finance », mis en ligne le 15 novembre 2008. URL :

http://regulation.revues.org/document5003.html>

BOUQUIN, H. (dir.) (2005), Les grands auteurs en contrôle de gestion, éditions EMS, collection Grands auteurs.

BOUSSARD, V., MAUGERI S. (dir.) (2003), Du politique dans les organisations, sociologie des dispositifs de gestion, L'Harmattan.

BURCHELL S., CLUBB C., HOPWOOD A., HUGHES J., NAHAPIET J. (1980), " The roles of Accounting in Organizations and Society ", Accounting, Organizations and Society, vol. 5, $\mathrm{n}^{\circ} 1, \mathrm{p} .5-27$.

BURLAUD, A., GIBERT, P. (1984), "L'analyse des coûts dans les organisations publiques : Le jeu et l'enjeu », Politiques et Management Public 2 (1): 93-117.

CHATELAIN-PONROY S. (2008), Le contrôle de gestion dans des bureaucraties professionnelles non lucratives: une proposition de modélisation, Habilitation à diriger des recherches, Université de ParisDauphine, 15 septembre.

CHATELAIN-PONROY S., SPONEM S. (2008), "Comme l'entreprise l'Etat doit adopter une culture du résultat ", in Petit bréviaire des idées reçues en management sous la direction d'A. Pezet et de S. Sponem, Edition La Découverte.

CHATY, L. (1999), « La 'responsabilisation' et le contrat managérial : figures et outils de la performance administrative en Europe ", Politiques et Management Public 17 (2): 85-103.

CHEMLA-LAFAY A., CHOL C. (2006), 25 ans de réforme de la gestion publique dans les pays de l'OCDE. Convergence et systémique, IGPDE, mai.

Club CAMBON (1988), Rapport introductif de la journée d'étude du 17 octobre 1987 du club Cambon sur l'évaluation des politiques publiques, Politiques et Management Public 6 (2) : 98-109. 
CROZIER M. (1963), Le Phénomène bureaucratique, Paris, Le Seuil.

CROZIER M. (1986), Etat modeste, Etat moderne. Stratégies pour un autre changement, Paris, Fayard.

GADREY, J. (1991), « Le service n'est pas un produit : Quelques implications pour l'analyse économique et pour la gestion », Politiques et Management Public 9 (1): 1-24.

GIBERT, P. (1986), « Management public, management de la puissance publique », Politiques et Management Public 4 (2) : 89-123.

GIBERT, P. (2000), « Mesure sur mesure », Politiques et Management Public 18 (4): 61-89.

GIBERT, P. (2002), « Réflexion sur l'utilisation du contrôle interne à des fins de contrôle externe ", Politiques et Management Public : 7-23.

HOFSTEDE G. (1981), « Management Control of Public and not-forProfit Activities, Accounting ", Organizations and Society, vol. $6, n^{\circ} 3$ : 93-211

JONCOUR, Y. (1987), « La régulation du service public hospitalier : Une 'greffe' des techniques du management privé ? ", Politiques et Management Public 5 (3): 117-132.

KNOEPFEL, P., VARONE, F. (1999), « Mesurer la performance publique: Méfions-nous des terribles simplificateurs », Politiques et Management Public 17 (2) : 123-145.

LAMBERT, A. (2002), Intervention au IVème forum de la gestion publique, Alain Lambert, ministre délégué au budget et à la réforme budgétaire.

MATHESON A., 30 ans de réforme de l'Etat, Dunod.

MONJARDET D. (2006), « Comment apprécier une politique policière ? Le premier ministère Sarkozy ", Sociologie du Travail, $n^{\circ} 48, p .188$ 208.

O.C.D.E. (2003), « La modernisation du secteur public », Synthèses OCDE, novembre.

O.C.D.E. (2005), « Evaluer les réformes », Observateur OCDE, novembre.

OSTERLOH M., FREY B., HOMBERG F. (2008), « Le chercheur et l'obligation de rendre des comptes ", traduit par H. DUMEZ, Gérer et Comprendre, $\mathrm{n}^{\circ} 91$, mars, p. 48-54.

OUCHI, W. G. (1979), «A conceptual framework for the design of organizational control mechanisms ", Management Science, 25 : 833848. 
PALLEZ, F. (2000), « De la mesure dans un service public régalien. Peut-on et faut-il quantifier la charge de travail des magistrats ? " Politiques et Management Public 18 (4) : 91-118.

PERRET, B. (2006), " De l'échec de la rationalisation des choix budgétaires (Rcb) à la loi relative aux lois de finances (Lolf) " Revue française d'administration publique 1 (117) : 31-41.

POLLITT C. (2002), " Convergence: the useful myth? " Public Administration, $79: 4$.

PORTER, T. M. (1995), Trust in numbers, Princeton, New Jersey: Princeton University Press.

ROBSON Keith (1992), "Accounting numbers as "inscriptions" : Action at a distance and the development of accounting ", Accounting, Organizations and Society, 17 (7), 685-708.

SEDDON, J. (2008), Systems Thinking in the Public Sector, Axminster (UK): Triarchy Press.

TROSA, S. (1992), « La modernisation est-elle évaluable ? », Politiques et Management Public 10 (4) : 65-83.

TROSA, S. (2000), « De la mesure à l'évaluation, de la performance à l'action, l'expérience d'une praticienne », Politiques et management public 18 (4) : 119-136.

WEICK E. Karl (1976), « Educational Organizations as Loosely Coupled Systems ", Administrative Science Quarterly, Vol. 21, №1 (March), pp. 1-19. 